\title{
Assessing Child Impairment in Functioning in Different Contexts: Implications for Use of Services and the Classification of Psychiatric Disorders
}

\author{
Glorisa J. Canino ${ }^{1}$, Prudence W. Fisher ${ }^{2}$, Margarita Alegria ${ }^{3}$, Héctor R. Bird ${ }^{2}$ \\ ${ }^{1}$ University of Puerto Rico, Medical Sciences Campus, San Juan, Puerto Rico \\ ${ }^{2}$ Columbia University, New York, USA \\ ${ }^{3}$ Harvard Medical School and Cambridge Health Alliance, Boston, USA \\ Email: glorisa.canino@upr.edu
}

Received November 1, 2012; revised December 5, 2012; accepted December 17, 2012

\begin{abstract}
Context: Functional impairment is a necessary criterion for most DSM IV disorders, for determining need for services, for evaluating the effectiveness of treatment and for reimbursement of mental health services. It is also an important predictor of mental health service utilization in children. Presently the diagnosis of impairment on axis V of DSM IV makes it difficult to disentangle symptoms and functioning. Furthermore, clinicians are required to consider familial and contextual issues in assessing child impairment, but no specific guidance or guidelines to accomplish this are offered. Conclusions: Given these limitations, a new conceptualization of impairment in functioning based on a modification of the International Classification of Functional Disability and Health for Children and Youth (ICF-CY) is recommended. A new instrument, the Child WHODAS, which is being considered for inclusion in the new edition of DSM 5, is described. This instrument is a disability measure based on the ICF-CY for ascertaining disability in functioning.
\end{abstract}

Keywords: Child Psychiatry; Measurement Development; Disability; Cross Cultural Child Behavior

\section{Introduction}

There are limited resources available for mental health service. Managed care payers attempt to restrict services to those who in their judgment "really" need them, thereby containing costs and increasing the companies' profit margins. To determine need managed care and insurance companies purportedly follow criteria established by Public Law 102 - 321 by which mandates the provision of mental health services only for children who have severe emotional disturbance (SED), defined as children who meet DSM IV diagnostic criteria [1] for mental disorders who also have substantial impairment in functioning [2]. Of significance is the fact that this legislation separates disability from psychopathology Thus, the classification of functional impairment (from now on called disability) associated with psychiatric disorder has become a necessary requirement for the reimbursement of mental health services and a necessary criterion for the allocation of resources.

In this paper we discuss several aspects of mental health disability in children including the functional disability requirement as an important construct for determining need for mental health services in children and for determining a DSM diagnosis, In addition we de- scribe a new instrument which is being considered for inclusion as a disability measure based on the International Classification of Functional Disability and Health for Children and Youth (ICF-CY) [3] for ascertaining disability in functioning in the new edition of DSM 5.

Central to the discussion of these issues is a clear and operational conceptualization of functional disability. We propose a definition based on the classificatory system of the ICF-CY, by which disability refers to decrements in bodily functions (both physiological and psychological) which are manifest at the individual level as activity limitations (difficulties the child may have at executing activities) and at the societal level as resulting in restrictions in participation or problems the child may have in typical life situations such as at school, or with peers, with his/her family and in the community at large [3].

\section{Importance of Ascertaining Functional Disability for Determining Need for Mental Health Services}

Since the advent of DSM III [4], the designation of a mental disorder has included disability or functional impairment in order for a disorder to be considered as present. However, clinicians and researchers have been lax 
in their consideration of this disability criterion when assigning diagnoses. Indeed, the very high rates of child psychiatric disorders (around 49\%) reported by many population based epidemiology studies that were based on the DSM III and DSM III R nosology have been held by many to be largely the result of not requiring any impairment or disability when considering caseness $[5,6]$. Concerns about these high rates of disorder likely the result of "false positives" led to a greater emphasis on disability (called impairment previously) in DSM IV which made impairment or distress an explicit requirement by including it as a separate and necessary criterion for many disorders [7]. As a result of this greater emphasis on disability most population based epidemiologic studies that used the DSM IV criteria observed much lower rates of child psychiatric disorder (ranging from $17 \%$ to $20 \%$ ) than had been reported previously [8-12].

In addition to the problem of inflated (perhaps unbelievable) rates of disorder when impairment or disability is not considered considerations on functional disability by both DSM IV and researchers is also critical because declines in functioning, unexpected behavioral deviations and disruptive behavior are the most common reasons that mental health services are first sought for children and adolescents [13-15]. Disability in functioning is more likely to lead parents to take their children into treatment than is a diagnosable psychiatric disorder $[9,16]$ and perception of disability appears to be more significant than diagnosis in predicting service utilization $[9,13]$. These results indicate that a caregiver's decision to seek care requires an awareness of the child's functioning.

It has also been reported that when a child meets criteria for more than one disorder, clinicians are more likely to treat the most impairing disorder first [17]. Moreover, prospective studies show that it is the level of disability and not symptom presentation that predicts adult outcomes [17]. Improvement in functioning is the main diagnostic outcome used for determining the effectiveness of any mental health intervention, and the litmus tests that society uses to evaluate the intervention. Identifying limitations in functioning in a child provides important information potentially useful for planning and implementing service interventions [18].

Thus, it appears fitting that a determination of "need for services" should rest substantially on the individual's level of functioning and disability. A child may experience significant symptoms of a psychiatric disorder that result in little or no functional disability. Conversely a child can have rather severe functional disability with relatively few symptoms, lending support to the notion that psychiatric symptoms and functional disability are two distinct domains of an individual's psychiatric presentation [19], of which clinical disability seems to be of greater clinical relevance. Several studies have shown that there is a group of children with significant disability that lack sufficient symptoms to meet symptom criteria for a specific disorder [20,21]. An emphasis on functional disability can result in early intervention for children at risk of significant morbidity and prevent delaying an intervention until after significant morbidity has occurred.

\section{The Need for a Measure for Ascertaining Functional Disability in the Forthcoming DSM 5}

While criteria for establishing the symptom criteria for a DSM IV disorder are well established [1], the decision of what constitutes substantial disability in social, occupational or other areas of functioning is variable and left up to the interpretation of the clinician or investigator using the manual. As noted, virtually no guidance was offered by DSM IV as to the operationalization of disability necessary to warrant a psychiatric diagnosis. In spite of the fact that research has demonstrated that symptoms and functional disability are clear-cut and distinct domains, in practice, the DSM combined symptoms and functioning in a way that made it difficult to disentangle the constructs.

As noted above, in DSM IV many diagnoses include an "impairment" criterion to assign diagnosis. However, this assumes that the clinician or patient (or in the case of children, the parent and/or child and/or teacher) can correctly attribute the cause of the patient's impairment as being the symptoms from the diagnosis being assigned. As it turns out, this is a difficult task. That is, is it the patient's depression that is causing him to not perform well at school OR might it be his anxiety or his attention deficit disorder? Bird et al. [22] found that it was difficult for children and their parents to make accurate attribution of impairment to specific symptoms in subjects who had comorbid diagnoses, if same impairment would be endorsed for each of the diagnoses. It is unclear at this point whether DSM 5 will keep or drop this criterion.

In addition to considering the impairment criterion included as one of the criteria for most disorders, when using the DSM IV TR [23] clinicians are asked to consider measuring children's impairment in functioning with the Child Global Assessment in Functioning (CGAS) provide an overall rating of impairment for Axis V, using the Global Assessment of Functioning Scale (GAF). For the GAF, the clinician picks a single number from 1 to 100 on a hypothetical continuum of mental healthillness to indicate overall functioning. This scale was adapted from the Global Assessment Scale (GAS, [24] and the corresponding child scale, the CGAS, adapted by Shaffer and colleagues [25], so that it could be used for all ages. The GAF requires clinicians to assess not only functioning in different life domains, (e.g., family, social, 
academic/occupational) but also includes psychological functioning which confounds functioning with the expression of psychiatric symptoms and behaviors (e.g., suicide attempts, manic excitement). Thus, we, agree with other investigators that the GAF is not an independent measure of disability $[26,27]$.

Further, the instructions for use of the scale offer no specific guidance as to how clinicians or investigators can accomplish this task, except that "functioning due to physical (or environmental) limitations should not be considered. This represents a challenge for both since the focus of the DSM was to describe psychiatric disorders and not to develop a classification of functional disability. On the other hand, the adult ICF [28] and youth ICF-CY [3] and were designed specifically as a classificatory system of disability and consequently provide the clinician with criteria that offer guidance for assessing and recording different types of disability and the contextual factors that might significantly contribute to the presentation, occurrence and outcome of mental or physical disabilities [29]. These systems recognize that "one size may not fit all" by having a separate system for youth. An example related to children would be code Z61.3 "Events resulting in loss of self-esteem in childhood" [30]. The implementation of the ICF-Y has been done mostly with physically or developmentally disabled children [31] however, and its applicability to children with mental disorders, has been very limited because of the lack of a valid tool to measure disability in children based on this classificatory system.

The major obstacle in developing appropriate measures of children's disability has been due, to a great extent, to the absence until recently [3] of an international classificatory system of impairment that could serve as foundation for the development of an impairment instrument. There was also the need to adapt the ICF-CY to make it applicable to children with mental health problems or disorders. A recent review of child measures of disability reported by Simeonsson and colleagues [31] demonstrated that most of these measures were not based on the current ICF-CY. Until now, the only measure based on the current ICF [28] was the World Health Organization Disability Assessment Schedule (WHODAS II) for adults [32,33], with no similar measure for children; in spite of the fact that an important requirement for research studies was the availability of assessment tools that empirically appraise disability.

\section{A New Instrument for Assessing Functional Disability for Children}

Like adults, children with chronic conditions such as severe emotional disturbance, vary greatly in their ability to perform in different contexts. Conversely, the contexts, culture and environment in which children's lives unfold affect their functioning [18]. The nature of a child's environment is different from those of adults: in early childhood, the child's environment is largely controlled by parents, so that child/caregiver's relationships and family environment are important components of the context in which the young child functions. As the child ages, school becomes an important context at this is where the child spends most of his/her days. Finally, as adolescence approaches both peers and the community at large become much more salient.

A new instrument to measure child disability, the Child WHODAS II, has been developed that contains domains related to these varying contexts and is based on the assumption that cultures and contexts vary and are important to consider in assessing functional disability. The Child WHODAS II was adapted for children from the adult WHODAS II [33] by the DSM 5 Impairment/ Disability workgroup [34] of which one of the authors (GC) of this paper belongs and another (PF) serves as an advisor. The adaptation process included making sure that the items could be well understood by children and their families, and that the items were consonant with the basic assumptions of child disability described in the ICF-CY [3].

The child WHODAS II conceptualizes functional disability as resulting not only from a medical or mental health condition inherent to the individual but from the interaction of the individual's health or mental health condition with environmental/contextual factors and changes related to developmental stage [31]. While the DSM IV required that the disability observed in the child resulted from the symptoms of the specific disorder, an attribution that many parents, particularly low education and minority parents, could not make reliably [35], in the ICF-CY and consequently in the child WHODAS II, functional disability is a separate construct from the disorder or illness and does not necessarily have to occur as a result of the same.

Furthermore, the conceptualization of the workgroup of disability in children as well as that of the ICF-CY takes into consideration that growth and functioning change rapidly in the developing child and that disabilities may be more difficult to ascertain than in adults particularly for young children who may lack the necessary cognitive development to serve as an informant of his/her disabilities [36]. For this reason, the child WHODAS II has two versions, one to be completed by parents about their children ages 0 to 17 and another parallel form for youth 12 years and above to complete about themselves. In addition, there is a clinician version. Consonant with the ICF-CY, the three versions of the child WHODAS assess the following domains: understanding and communicaing, getting around (mobility), self care, getting along 
with people, life activities (school and non-school), participating in society. The time frame for all three versions is the last 30 days.

Parent and Youth Version: The parent and the youth versions begin with a global rating of overall health in the past 30 days, rated on a five point scale (very good, good, moderate, bad, and very bad). This is followed by 34 items divided across the different domains as follows: understanding and communicating-6 items; getting around -5 items; self care -4 items; getting along with people- 5 items; life activities-4 items for non-school and 5 items for school; participation in society 5 items. For each the respondent considers the level of difficulty on a five point scale (none, mild, moderate, severe, extreme/cannot do). At the end of the questionnaire the participant is asked to provide an overall rating of how much his/her difficulties interfered with the child's life (using the same five point scale), the number of days (or the last thirty) the difficulties were present, the child was unable to carry out usual activities or the child had to cut back on usual activities, how many days the child was late for school and missed school and number of days absent from school and late to school.

Clinician Version: The clinician version also begins with an overall rating of the patient's health rated on a five point scale ranging from very good to very bad. This is followed by 7 items, one for each of the domains spelt out above on which the clinician makes an overall rating as to level of difficulty using the five point "none" to "extreme/cannot do" scale described above. Two additional ratings about how much the difficulties caused interference in the patient's (or family's) life and how much the difficulties had been a drain of the financial resources of the family are also made by the clinician.

\section{Conclusions and Recommendations for Future Research}

The present proposal of the DSM 5 impairment workgroup is to substitute the GAF recommended by the DSM-IV-TR [23] with the child WHODAS II in order to assess disability in children. The test-retest reliability of the new instrument and its acceptability and usefulness for clinical planning is presently being tested as part of the DSM 5 field trial, of up to 1000 child and adolescent patients being carried out by four academic centers in the mainland United States. There is a need though to test the psychometrics of the instrument in other cultures and contexts different from the United States mainland.

In summary, classification systems and measures of disability should provide a basis for determining need for services, providing appropriate treatment interventions, addressing parental concerns and providing equity in the allocation of services. We feel that the Child WHODAS
II has the potential to achieve these goals, particularly if future studies test the psychometric properties of the instruments in other cultures and contexts. However, classification is a tool and its usefulness or lack there of depends for whom and under what circumstances the measure is used. The recognition of researchers, educators and policymakers of the need to use a measure based on a classification of disability within the ICF-CY model that takes into consideration individual needs in interacttion with contextual, family and cultural factors is important to avoid stigmatizing, labeling, and under or over diagnosing children with disabilities [36].

Cost-containment strategies will be emphasized and enforced by payers during the next decade, and greater demand for children's disability measures will come into effect. Having explicit criteria that facilitate identifying children in terms of disability and adjustment will be critical. Making sure that we have a global rather than an ethnocentric perspective in our measurement and criteria development is crucial.

\section{REFERENCES}

[1] American Psychiatric Association, "Diagnostic and Statistical Manual of Mental Disorders," 4th Edition, American Psychiatric Association, Washington DC, 1994.

[2] USA Department of Health and Human Services, "Substance Abuse and Mental Health Services Administration (Notices),” Federal Register, Vol. 2, No. 58, 1993, p. 15.

[3] World Health Organization, "International Classification of Impairments, Disabilities, and Handicaps-Version for Children \& Youth,” World Health Organization, Geneva, 2007.

[4] American Psychiatric Association, "Diagnostic and statistical Manual of Mental Disorders,” 3rd Edition, American Psychiatric Association, Washington DC, 1980.

[5] H. R. Bird, T. J. Yager, B. Staghezza, M. S. Gould, G. Canino and M. Rubio-Stipec, "Impairment in the Epidemiological Measurement of Childhood Psychopathology in the Community," Journal of the American Academy of Child and Adolescent Psychiatry, Vol. 29, No. 5, 1990, pp. 796-803.

[6] G. Canino, E. J. Costello and A. Angold, “Assessing Functional Impairment and Social Adaptation for Child Mental Health Services Research: A Review of Measures," Mental Health Services and Research,Vol. 1, No. 2, 1999, pp. 93-108. doi:10.1023/A:1022334303731

[7] R. L. Spitzer and J. C. Wakefield, "DSM-IV Diagnostic Criterion for Clinical Significance: Does it Help Solve the False Positives Problem?” American Journal of Psychiatry, Vol. 156, No. 12, 1999, pp.1856-1864.

[8] H. Bird, M. Davies, C. S. Duarte, S. Shen, R. Loeber and G. J. Canino, “A Study of Disruptive Behavior Disorders in Puerto Rican Youth: II . Baseline Prevalence, Comorbidity, and Correlates in Two Sites," Journal of the American Academy of Child and Adolescent Psychiatry, 
Vol. 45, No. 9, 2006, pp. 1042-1053. doi:10.1097/01.chi.0000227879.65651.cf

[9] G. Canino, et al., "The DSM-IV Rates of Child and Adolescent Disorders in Puerto Rico: Prevalence, Correlates, Service Use, and the Effects of Impairment," Archives of General Psychiatry, Vol. 61, No. 1, 2004, pp. 85-93.

[10] B. Fleitlich-Bilyk and R. Goodman, "Prevalence of Child and Adolescent Psychiatric Disorders in Southeast Brazil," Journal of the American Academy of Child and Adolescent Psychiatry, Vol. 43, No. 6, 2004, pp. 727-734. doi:10.1097/01.chi.0000120021.14101.ca

[11] T. Ford, R. Goodman and H. Meltzer, "The British Child and Adolescent Mental Health Survey 1999: The prevalence of DSM-IV disorders," Journal of the American Academy of Child and Adolescent Psychiatry, Vol. 42, No. 10, 2003, pp. 1203-1211.

[12] R. Goodman, H. Slobodskaya and G. Knyazev, "Russian Child Mental Health-A Cross-Sectional Study of Prevalence and Risk Factors,” European Child \& Adolescent Psychiatry, Vol. 14, No. 1, 2005, pp. 28-33. doi:10.1007/s00787-005-0420-8

[13] M. Alegría, et al., "Cultural Relevance and Equivalence in the NLAAS Instrument: Integrating Etic and Emic in the Development of Cross Cultural Measures for a Psychiatric Epidemiology and Services Study of Latinos,” International Journal of Methods in Psychiatric Research, Vol. 13, No. 4, pp. 270-288. doi:10.1002/mpr.181

[14] M. J. Briggs-Gowan, S. M. C. Horwitz, M. E. SchwabStone, J. M. Leventhal and P. J. Leaf, "Mental Health in Pediatric Settings: Distribution of Disorders and Factors Related to Service Use," Journal of the American Academy of Child and Adolescent Psychiatry,Vol. 39, No. 7, 2000, pp. 841-849.

[15] S. P. Cuffe, et al., "A Longitudinal Study of Adolescent Mental Health Service Use,” Journal of Behavioral Health and Services Research, Vol. 28, No. 1, 2001, pp. 1-11. doi:10.1007/BF02287230

[16] A. M. Chronis, et al., "Comprehensive, Sustained Behavioral and Pharmacological Treatment for AttentionDeficit/Hyperactivity Disorder: A Case Study,” Cognitive and Behavioral Practicem, Vol. 8, No. 4, 2001, pp 346359. doi:10.1016/S1077-7229(01)80008-0

[17] M. Gordon, et al., "Symptoms versus Impairment: The Case for Respecting DSM-IV's Criterion D,” Journal of Attention Disorders, Vol. 9, No. 3, 2006, pp. 465-475.

[18] D. J. Lollar and R. J. Simeonsson, "Diagnosis to Function: Classification for Children and Youths," Journal of Developmental and Behavioral Pediatrics, Vol. 26, No. 4, 2005, pp. 323-330. doi:10.1097/00004703-200508000-00012

[19] A. F. Lehman, G. S. Alexopoulos, H. Goldman, M. D. Dilipjeste and B. Ustün, "Mental Disorders and Disability: Time to Reevaluate the Relationship?” In: D. J. Kupfer, M. B. First, and D. A. Regierm, Eds., A Research Agenda for DSM-V, American Psychiatric Press Inc., Washington DC, 2002, pp. 201-218.

[20] A. Angold, E. J. Costello, E. M. Z. Farmer, B. J. Burns and A. Erkanli, "Impaired but Undiagnosed," Journal of the American Academy of Child and Adolescent Psychiatry, Vol. 38, No. 2, 1999, pp. 129-137.

[21] J. Biederman, E. Mick and S. V. Faraone, “Age-Dependent Decline of Symptoms of Attention Deficit Hyperactivity Disorder: Impact of Remission Definition and Symptom Type," American Journal of Psychiatry, Vol. 157, No. 5, 2000, pp. 816-818. doi:10.1176/appi.ajp.157.5.816

[22] H. R. Bird, et al., "The Columbia Impairment Scale (CIS): Pilot Findings on a Measure of Global Impairment for Children and Adolescents," International Journal of Methods in Psychiatric Research, Vol. 3, No. 3, 1993, pp. 167-176.

[23] American Psychiatric Association, "Diagnostic and Statistical Manual of Mental Disorders," 4th Edition, American Psychiatric Association, Washington DC, 2000.

[24] J. Endicott, R. L. Spitzer, J. L. Fleiss and J. Cohen, “The Global Assessment Scale. A Procedure for Measuring Overall Severity of Psychiatric Disturbance, Archives of General Psychiatry, Vol. 33, No. 6, 1976, pp. 766-771. doi:10.1001/archpsyc.1976.01770060086012

[25] D. Shaffer, et al., "A Children’s Global Assessment Scale (CGAS),” Archives of General Psychiatry, Vol. 40, No. 11, 1983, pp. 1228-1231. doi:10.1001/archpsyc.1983.01790100074010

[26] W. K. Goodman, E. A. Storch, G. R. Geffken and T. K. Murphy, "Obsessive-Compulsive Disorder in Tourette Syndrome,” Journal of Child Neurology, Vol. 21, No. 8, 2006, pp. 704-714. doi:10.1177/08830738060210081201

[27] B. Green, S. Shirk, D. Hanze and J. Wanstrath, "The Children's Global Assessment Scale in Clinical Practice: An Empirical Evaluation,” Journal of the American Academy of Child and Adolescent Psychiatry, Vol. 33, No. 8, 1994, pp. 1158-1164.

[28] World Health Organization, "International Classification of Functioning, Disability and Health (ICF),” World Health Organization, Geneva, 2001.

[29] A. Janca, M. C. Kastrup, H. Katschnig, J. J. Lopez-Ibor, J. E. Mezzich and N. Sartorius, "The ICD-10 Multiaxial System for Use in Adult Psychiatry: Structure and Applications,” Journal of Nervous and Mental Disease, Vol. 184, No. 3, 1996, pp. 191-192. doi:10.1097/00005053-199603000-00010

[30] T. B. Ustün, "Using the International Classification of Functioning, Disease and Health in Attention-Deficit/Hyperactivity Disorder: Separating the Disease from Its Epiphenomena," Ambulatory Pediatrics, Vol. 7, No. 1, 2007, pp. 132-139. doi:10.1016/j.ambp.2006.05.004

[31] R. J. Simeonsson, M. Leonardi, D. Lollar, E. BjorckAkesson, J. Hollenweger and A. Martinuzzi, “Applying the International Classification of Functioning, Disability and Health (ICF) to Measure Childhood Disability,” Disability and Rehabilitation, Vol. 25, No. 11-12, 2003, pp. 602-610

[32] World Health Organization, "WHO Disability Assessment Schedule (WHODAS II) Training Manual: A Guide to Administration," World Health Organization, Geneva, 2000.

[33] M. Von Korff, et al., "Modified WHODAS- II Provides 
Valid Measure of Global Disability but Filter Items Increased Skewness," Journal of Clinical Epidemiology, Vol. 61, No. 11, 2008, pp. 1132-1143.

[34] National Institute of Mental Health, “Conceptualization and Measurement of Functioning in Children or Adolescents with or at Risk for Mental Disorder,” National Institute of Mental Health, Bethesda, 2005.
[35] H. R. Bird, et al., "How Specific Is Specific Impairment?" Journal of the American Academy of Child and Adolescent Psychiatry, Vol. 39, No. 9, 2000, pp.11821189. doi:10.1097/00004583-200009000-00019

[36] L. Florian, et al., "Cross-Cultural Perspectives on the Classification of Children with Disabilities," Journal of Special Education, Vol. 40, No. 1, 2006, pp. 36-45. 